\title{
Adaptive Attitude Control for a Small Satellite with Integrated Singularity Avoidance and Momentum Management
}

\author{
D. Kim ${ }^{1}$, F. A. Leve ${ }^{2}$, N. Fitz-Coy ${ }^{1}$, and W. E. Dixon ${ }^{1}$ \\ ${ }^{1}$ Mechanical and Aerospace Engineering Department, University of Florida, Gainesville, FL 32611-6250, US \\ ${ }^{2}$ Air Force Research Lab, Albuquerque, NM 87117, US \\ Email: \{ndsatellite,nfc,wdixon\}@ufl.edu, afrl.rvsv@kirtland.af.mil
}

\begin{abstract}
An adaptive attitude controller for a small satellite utilizing variable speed control moment gyroscopes (VSCMGs) is proposed, which performs integrated singularity avoidance and momentum management using null motion. An adaptive controller yields accurate attitude tracking while the VSCMG null motion achieves singularity avoidance and momentum management. The proposed controller compensates for parametric uncertainties present in the nonlinear satellite dynamics. Asymptotic attitude tracking and exponential momentum tracking are proven via Lyapunov stability analysis. Simulation results indicate the performance of the proposed controller as a VSCMG steering law.
\end{abstract}

\section{INTRODUCTION}

Research on the control of variable speed control moment gyroscopes (VSCMGs) has shown up in literature for about the last twenty years [1]-[4]. Integrated Power and Attitude Control System (IPACS) and/or Flywheel Attitude Control and Energy Transmissions Systems (FACETS) use VSCMGs for power storage by de-spinning the flywheels for the deceleration mode of the VSCMG and absorbing their kinetic energy [5]-[9]. An adaptive attitude controller for a VSCMGbased satellite is proposed where attitude control torques are generated by means of a pyramidal arrangement of four single gimbal VSCMGs. The proposed controller functioning as a VSCMG steering law is developed in terms of the gimbal rates and the flywheel accelerations which are weighted by the singularity measure to actively exploit the benefit of VSCMGs which has the additional degree of freedom. Using null motion, a strategy is developed to simultaneously perform the gimbal reconfiguration for internal singularity avoidance and the wheel speed regularization for reduced power and external singularity avoidance. Responding to the singularity measure, the gimbal reconfiguration contributes to internal singularity avoidance and the wheel regularization redistributes momentum to the spacecraft for attitude control in a way that reduces the total power to control the system of VSCMGs. Lyapunov stability analysis was performed to ensure that the controller is capable of achieving globally asymptotically attitude tracking and exponential momentum tracking. A simulation, staring at elliptic singularity, was performed to demonstrate the performance of the composite adaptive VSCMG steering law to demonstrate the efficacy of singularity avoidance with reduced reaction wheel modes and the reduction of input power for control of VSCMGs. Furthermore, to evaluate the performance of the proposed controller in a severe situation, a sudden disturbance or task assignment, modeled as a smooth s-function, was executed during the simulation. The simulation demonstrates that the controller was capable of handling these severe disturbances (e.g., significant increases in friction due to foreign object debris). In summary the nonlinear adaptive controller compensates for inertia uncertainties while reducing the power needed for control and maintaining stability. The proposed controller has a combined form of the gimbal angular velocity and the flywheel acceleration rather than a control torque to solve the attitude tracking problem, therefore the development has complication by the fact that the control input is multiplied by a time-varying, nonlinear uncertain matrix. Sections II and III develop the dynamic and kinematic models of the system. The overall control objectives are described in section IV and a detailed analysis of the adaptive VSCMG steering law is presented in section V. Simulation results in Section VI illustrate performance of the proposed adaptive attitude controller as a VSCMG steering law. Conclusions are presented in section VII.

\section{DyNAMiC MODEL}

The dynamic model for a rigid satellite with a pyramid configuration of four VSCMG units can be expressed as [10]

$$
\begin{aligned}
J \dot{\omega}+\dot{J} \omega+\omega^{\times} J \omega= & -A_{g} I_{c g} \ddot{\delta}-A_{t} I_{w s}[\Omega]^{d} \dot{\delta} \\
& -\omega^{\times} A_{g} I_{c g} \dot{\delta}-A_{s} I_{w s} \dot{\Omega} \\
& -\omega^{\times} A_{s} I_{w s} \Omega .
\end{aligned}
$$

In (1), $\omega(t), \dot{\omega}(t) \in \mathbb{R}^{3}$ denote the angular velocity and acceleration of the satellite body-fixed frame $\mathcal{F}$. with respect to the inertial frame $\mathcal{I}$ expressed in $\mathcal{F}, \Omega(t), \dot{\Omega}(t) \in \mathbb{R}^{4}$ are the angular rate and acceleration of the VSCMG wheels, and $\delta(t), \dot{\delta}(t), \ddot{\delta}(t) \in \mathbb{R}^{4}$ are the angle, rate, and acceleration of the VSCMG gimbals. The uncertain total spacecraft inertia matrix $J(\delta) \in \mathbb{R}^{3 \times 3}$ is positive definite and symmetric such that

$$
\frac{1}{2} \lambda_{\min }\{J\}\|\xi\|^{2} \leq \xi^{T} J \xi \leq \frac{1}{2} \lambda_{\max }\{J\}\|\xi\|^{2} \quad \forall \xi \in \mathbb{R}^{n}
$$

where $\lambda_{\min }\{J\}, \lambda_{\max }\{J\} \in \mathbb{R}$ are the minimum and maximum eigenvalues of $J(\delta)$, respectively. In (1), $I_{c g} \in \mathbb{R}^{4 \times 4}$ is an uncertain constant diagonal matrix whose elements are the centroidal inertia components of each VSCMG assembly 
about its gimbal direction, $I_{w s} \in \mathbb{R}^{4 \times 4}$ denotes a known positive-definite symmetric flywheel inertia matrix about its spin axis, $[\Omega]^{d}$ is the diagonal matrix, and $\omega^{\times}$is the skewsymmetric matrix defined as

$$
\omega^{\times}=\left[\begin{array}{ccc}
0 & -\omega_{3} & \omega_{2} \\
\omega_{3} & 0 & -\omega_{1} \\
-\omega_{2} & \omega_{1} & 0
\end{array}\right] .
$$

Also in (1), the measurable matrices $A_{g}, A_{s}(\delta), A_{t}(\delta) \in$ $\mathbb{R}^{3 \times 4}$ ensure the gimbal, spin, and transverse inertia components of each flywheel/gimbal assembly about its gimbal, spin, and transverse direction are transformed to the satellite body-fixed frame.

\section{KinematiC MODEL}

The rotational kinematics of the rigid-body satellite can be expressed as

$$
\begin{gathered}
\dot{q}_{v}=\frac{1}{2}\left(q_{v}^{\times} \omega+q_{0} \omega\right) \\
\dot{q}_{0}=-\frac{1}{2} q_{v}^{T} \omega .
\end{gathered}
$$

In (3) and (4), $q(t) \triangleq\left\{q_{0}(t), q_{v}(t)\right\} \in \mathbb{R} \times \mathbb{R}^{3}$ represents the unit quaternion describing the orientation of the body-fixed frame $\mathcal{F}$ with respect to $\mathcal{I}$, subject to the constraint

$$
q_{v}^{T} q_{v}+q_{0}^{2}=1
$$

Using (3) and (4), $\omega(t)$ can be expressed in terms of the quaternion as

$$
\omega=2\left(q_{0} \dot{q}_{v}-q_{v} \dot{q}_{0}\right)-2 q_{v}^{\times} \dot{q}_{v} .
$$

The desired angular velocity of the body-fixed frame $\mathcal{F}_{d}$ with respect to $\mathcal{I}$ expressed in $\mathcal{F}_{d}$ can also be determined as

$$
\omega_{d}=2\left(q_{0 d} \dot{q}_{v d}-q_{v d} \dot{q}_{0 d}\right)-2 q_{v d}^{\times} \dot{q}_{v d} .
$$

The subsequent analysis is based on the assumption that the desired quaternion $q_{v d}(t), q_{0 d}(t)$, and the respective first three time derivatives are bounded for all time. This assumption ensures that $\omega_{d}(t)$ of (7) and its first two time derivatives are bounded for all time.

\section{Control Objective}

\section{A. Attitude Control Objective}

The attitude control objective is to develop a flywheel acceleration and gimbal rate control law to enable the attitude of $\mathcal{F}$ to track the attitude of $\mathcal{F}_{d}$. An attitude tracking error denoted by $\tilde{R}\left(e_{v}, e_{0}\right) \in \mathbb{R}^{3 \times 3}$ can be stated as

$$
\tilde{R}\left(e_{v}(t), e_{0}(t)\right) \rightarrow I_{3} \quad \text { as } \quad t \rightarrow \infty .
$$

The open-loop quaternion tracking error is given as

$$
\dot{e}_{v}=\frac{1}{2}\left(e_{v}^{\times}+e_{0} I\right) \tilde{\omega} \quad \dot{e}_{0}=-\frac{1}{2} e_{v}^{T} \tilde{\omega} .
$$

Based on the tracking error formulation, the angular velocity of $\mathcal{F}$ with respect to $\mathcal{F}_{d}$ expressed in $\mathcal{F}$, denoted by $\tilde{\omega}(t) \in$ $\mathbb{R}^{3}$, is defined as

$$
\tilde{\omega} \triangleq \omega-\tilde{R} \omega_{d}
$$

From the definitions of the quaternion tracking error variables, the following constraint can be developed:

$$
e_{v}^{T} e_{v}+e_{0}^{2}=1
$$

where

$$
0 \leq\left\|e_{v}(t)\right\| \leq 1 \quad 0 \leq\left|e_{0}(t)\right| \leq 1
$$

where $\|\cdot\|$ represents the standard Euclidean norm. From (11),

$$
\left\|e_{v}(t)\right\| \rightarrow 0 \Rightarrow\left|e_{0}(t)\right| \rightarrow 1
$$

and hence, the control objective in (8) will be achieved if (13) is satisfied.

\section{B. Flywheel Angular Momentum Tracking Objective}

The angular momentum tracking control objective is to develop a flywheel accleration control law so that the actual angular momentum $h(t)=I_{w s} \Omega(t)$ tracks a preferred constant angular momentum $h_{f}=I_{w s} \Omega_{f}$ while simultaneously tracking a desired time-varying attitude. To quantify the momentum tracking objective, an angular momentum tracking error $\mu(t) \in \mathbb{R}^{4}$ is defined as

$$
\mu=h_{f}-h(t) .
$$

In addition to the simultaneous desire to achieve attitude tracking, the momentum tracking is achieved in the null space of the VSCMGs.

\section{Development of Adaptive VSCMGs CONTROLLER}

\section{A. Adaptive Attitude Control Development}

To facilitate the control design, an auxiliary signal $r(t) \in$ $\mathbb{R}^{3}$ is defined as [11]

$$
r \triangleq \omega-\tilde{R} \omega_{d}+\alpha e_{v}
$$

where $\alpha \in \mathbb{R}^{3 \times 3}$ is a constant, positive definite, diagonal control gain matrix. After substituting (15) into (10), the angular velocity tracking error can be expressed as

$$
\tilde{\omega}=r-\alpha e_{v} .
$$

Motivation for the design of $r(t)$ is obtained from the subsequent Lyapunov-based stability analysis. After taking the time derivative of (15) and multiplying both sides of the resulting expression by $J(\delta)$, the following expression can be obtained:

$$
J \dot{r}=J \dot{\omega}+J \omega^{\times} \tilde{R} \omega_{d}-J \tilde{R} \dot{\omega}_{d}+\frac{1}{2} J \alpha\left(e_{v}^{\times}+e_{0} I\right) \tilde{\omega},
$$

where the fact that

$$
\dot{\tilde{R}}=-\omega^{\times} \tilde{R}
$$

was utilized. After substituting $\dot{\omega}(t)$ of (17) into (1), the expression in (1) can be rewritten as

$$
\begin{aligned}
J \dot{r}= & \Upsilon_{1} \dot{\delta}-A_{t} I_{w s}[\Omega]^{d} \dot{\delta}-A_{s} I_{w s} \dot{\Omega}+Y_{2} \theta_{2} \\
& -\omega^{\times} A_{s} I_{w s} \Omega-\frac{1}{2} \dot{J} r
\end{aligned}
$$


where the uncertain matrix $\Upsilon_{1}(\omega, \delta) \in \mathbb{R}^{3 \times 4}$ can be redefined via the parameterization

$$
\Upsilon_{1} \dot{\delta} \triangleq-\frac{\partial J}{\partial \delta} \dot{\delta}\left(\frac{1}{2} r+\tilde{R} \omega_{d}-\alpha e_{v}\right)-\omega^{\times} A_{g} I_{c g} \dot{\delta} .
$$

To compensate for the linearly parametrizable uncertainty present in $\Upsilon_{1}\left(e_{v}, r, \omega, \omega_{d}, \delta\right)$, the quantity $\Upsilon_{1} \dot{\delta}$ is reparametrized in terms of a known regression matrix $Y_{1}\left(e_{v}, r, \omega, \omega_{d}, \delta, \dot{\delta}\right) \in R^{3 \times p_{1}}$ and a vector of $p_{1}$ unknown constants $\theta_{1} \in R^{p_{1}}$ as

$$
Y_{1} \theta_{1} \triangleq \Upsilon_{1} \dot{\delta}
$$

To address the fact that the control input $\dot{\delta}(t)$ is premultiplied by the nonsquare, time-varying uncertain matrix $\Upsilon_{1}(\cdot)$, an estimate of the uncertainty in (20), denoted by $\hat{\Upsilon}_{1}(t) \in$ $R^{3 \times 4}$, is defined as

$$
Y_{1} \hat{\theta}_{1} \triangleq \hat{\Upsilon}_{1} \dot{\delta}
$$

where $\hat{\theta}_{1}(t) \in R^{p_{1}}$ is a subsequently designed estimate for the parametric uncertainty in $\Upsilon_{1}(\cdot)$. Also in (18), $Y_{2}\left(e_{v}, e_{0}, r, \omega, \omega_{d}, \dot{\omega}_{d}, \delta, \ddot{\delta}, \Omega\right) \theta_{2}$ is defined via the parameterization as

$$
\begin{aligned}
Y_{2} \theta_{2} \triangleq & -\omega^{\times} J \omega-A_{g} I_{c g} \ddot{\delta}+J \omega^{\times} \tilde{R} \omega_{d} \\
& -J \tilde{R} \dot{\omega}_{d}+\frac{1}{2} J \alpha\left(e_{v}^{\times}+e_{0} I\right) \tilde{\omega} .
\end{aligned}
$$

In (22), $Y_{2}(\cdot) \in \mathbb{R}^{3 \times p_{2}}$ is a measurable regression matrix, and $\theta_{2} \in \mathbb{R}^{p_{2}}$ is a vector of unknown constants. Based on (19) and (21), the expression in (18) can be rewritten as

$$
\begin{aligned}
J \dot{r}= & \hat{\Upsilon}_{1} \dot{\delta}-A_{t} I_{w s}[\Omega]^{d} \dot{\delta}-A_{s} I_{w s} \dot{\Omega}+Y_{1} \tilde{\theta}_{1} \\
& +Y_{2} \theta_{2}-\omega^{\times} A_{s} I_{w s} \Omega-\frac{1}{2} \dot{J} r
\end{aligned}
$$

where the notation $\tilde{\theta}_{1}(t) \in \mathbb{R}^{p_{1}}$ is defined as

$$
\tilde{\theta}_{1}=\theta_{1}-\hat{\theta}_{1} \text {. }
$$

To facilitate the subsequent development, (23) is rewritten as

$$
J \dot{r}=\hat{Q} \dot{\eta}+Y_{1} \tilde{\theta}_{1}+Y_{2} \theta_{2}-\omega^{\times} A_{s} I_{w s} \Omega-\frac{1}{2} \dot{J} r,
$$

where the torque rendered by the VSCMGs can be redesigned as

$$
\hat{Q} \dot{\eta}=-A_{s} I_{w s} \dot{\Omega}+\hat{\Upsilon}_{1} \dot{\delta}-A_{t} I_{w s}[\Omega]^{d} \dot{\delta},
$$

where $\dot{\eta}(t)=\left[\begin{array}{ll}\dot{\Omega}^{T} & \dot{\delta}^{T}\end{array}\right]^{T} \in R^{8 \times 1}$ is premultiplied by a nonsquare, time-varying uncertain matrix, an estimate of the uncertainty in (26), represented by $\hat{Q} \in R^{3 \times 8}$. Based on the expression in (25) and the subsequent stability analysis, the weighted adaptive attitude controller is designed as

$$
\begin{aligned}
\dot{\eta}= & -\hat{Q}_{w}^{+}\left(Y_{2} \hat{\theta}_{2}+e_{v}+k r-\omega^{\times} A_{s} I_{w s} \Omega\right) \\
& -\left(I_{8}-\hat{Q}_{w}^{+} \hat{Q}\right) S \sigma,
\end{aligned}
$$

where $\hat{Q}_{w}^{+}=W \hat{Q}^{T}\left(\hat{Q} W \hat{Q}^{T}\right)^{-1}$ and $W$ is a weight matrix determining whether the VSCMG system uses a CMG mode or a RW (Reaction Wheel) mode designed as [2], [12]

$$
W \triangleq\left[\begin{array}{cc}
W_{\Omega} I_{4 \times 4} & 0_{4 \times 4} \\
0_{4 \times 4} & W_{\delta} I_{4 \times 4}
\end{array}\right],
$$

where $W_{\Omega}$ is defined as

$$
W_{\Omega} \triangleq W_{\Omega 0} \exp \left(\lambda_{1} f\right),
$$

where $\lambda_{1}, W_{\Omega 0}, W_{\delta} \in \mathbb{R}$ are positive constants and the objective function $f$ measuring singularity can be denoted as

$$
f=-\operatorname{det}\left(A_{t} A_{t}^{T}\right) .
$$

In (27), the second term $\left(I_{8}-\hat{Q}_{w}^{+} \hat{Q}\right) S \sigma$ generates the VSCMG null motion for an efficient momentum management and gimbal reconfiguration, and $k \in \mathbb{R}$ is a positive control gain. Since the matrices $\hat{Q}_{w}^{+}$and $\hat{Q}$ are nonsquare, the pseudo-inverse $\hat{Q}_{w}^{+} \in \mathbb{R}^{8 \times 3}$ is defined so that $\hat{Q} \hat{Q}_{w}^{+}=I_{3}$, and the matrix $I_{8}-\hat{Q}_{w}^{+} \hat{Q}$, which projects vectors onto the null space of $\hat{Q}$, satisfies the properties

$$
\begin{aligned}
\left(I_{8}-\hat{Q}_{w}^{+} \hat{Q}\right)\left(I_{8}-\hat{Q}_{w}^{+} \hat{Q}\right) & =I_{8}-\hat{Q}_{w}^{+} \hat{Q} \\
\hat{Q}\left(I_{8}-\hat{Q}_{w}^{+} \hat{Q}\right) & =0 .
\end{aligned}
$$

To generate null motion for momentum tracking and gimbal reconfiguration, the null motion $\sigma(t) \in \mathbb{R}^{8 \times 1}$ is defined as

$$
\sigma \triangleq\left[\begin{array}{c}
k_{w} g \\
k_{\gamma} \frac{\partial \gamma}{\partial \delta}
\end{array}\right]
$$

where $k_{w}, k_{\gamma} \in \mathbb{R}$ are positive constants and $g(t)$ is a subsequently designed auxiliary control signal to track the desired flywheel angular momentum. The second row in (31) allows the null motion to perform the gimbal reconfiguration corresponding to variation of singularity measure index $\gamma$, which is defined as [13]

$$
\gamma=\gamma_{0} \exp \left(\lambda_{2} f\right)
$$

where the objective function $f$ is denoted in (29) and $\lambda_{2}, \gamma_{0} \in \mathbb{R}$ are positive constants. A matrix $S(\delta) \in \mathbb{R}^{8 \times 8}$ is used as a weight matrix for the VSCMG null motion, which can select a proper mode based on the singularity measure. Specifically, $S(\delta)$ is designed as

$$
\begin{aligned}
S & \triangleq \operatorname{diag}\left(\left[s_{w}, s_{g}\right]\right) \\
& =\left[\begin{array}{cc}
\operatorname{sech}\left(\frac{1}{k_{d} \operatorname{det}\left(A_{t} A_{t}^{T}\right)+\varepsilon}\right) & 0_{4 \times 4} \\
0_{4 \times 4} & \operatorname{sech}\left(k_{g} \operatorname{det}\left(A_{t} A_{t}^{T}\right)\right)
\end{array}\right],
\end{aligned}
$$

where $k_{g}, k_{d}, \varepsilon \in \mathbb{R}$. In (33), $s_{w}, s_{g} \in \mathbb{R}^{4 \times 4}$ weight either the momentum tracking or the gimbal reconfiguration corresponding to how approximate or far the $\mathrm{CMG}$ configuration is to a singularity. When the CMG configuration is singular, $S \approx \operatorname{diag}([0,1])$ and $S \approx \operatorname{diag}([1,0])$ otherwise. In the former case, the VSCMGs can be operated in RW mode corresponding to the weight of (28) and issued to enable the null motion of CMGs to avoid the singularity using 
the gimbal null motion (i.e., gimbal reconfiguration). In the latter case, the VSCMGs can track the desired momentum by generating the wheel null motion according to the momentum tracking objective (i.e., momentum tracking or wheel speed regularization). The control input $\dot{\eta}(t)$ in (27) is partitioned as

$$
\left[\begin{array}{c}
\dot{\Omega} \\
\dot{\delta}
\end{array}\right]=\left[\begin{array}{l}
-R_{1}-k_{w} N_{1} \bar{s}_{w} g \\
-R_{2}-k_{\gamma} N_{2} \bar{s}_{g} \frac{\partial \gamma}{\partial \delta}
\end{array}\right],
$$

where $R_{i}=\left[\hat{Q}_{w}^{+}\left(Y_{2} \hat{\theta}_{2}+e_{v}+k r-\omega^{\times} A_{s} I_{w s} \Omega\right)\right]_{i}$ indicates components of each $(4 \times 1)$ control input. Also in (34), $N_{1}, N_{2}$ are denoted as

$$
N=\left[\begin{array}{l}
{\left[N_{1}\right]_{4 \times 8}} \\
{\left[N_{2}\right]_{4 \times 8}}
\end{array}\right],
$$

and $\bar{s}_{w}, \bar{s}_{g}$ are also given as

$$
\bar{s}_{w}=\left[\begin{array}{ll}
s_{w} & 0_{4 \times 4}
\end{array}\right]^{T} \quad \bar{s}_{g}=\left[\begin{array}{ll}
0_{4 \times 4} & s_{g}
\end{array}\right]^{T} .
$$

In (35), $\left[N_{i}\right] \forall i=1,2$ is each upper and lower $(4 \times 8)$ matrix where $N_{i}=\left[\left(I-Q_{w}^{+} Q\right)\right]_{i} \forall i=1,2$ are null components, and each $s_{d}, s_{g}$ of switch matrix $S$ of (33) has $(4 \times 4)$ dimension. After substituting (27) into (25) and using the property (30b), the following closed-loop dynamics for $r(t)$ are obtained:

$$
J \dot{r}=-\frac{1}{2} \dot{J} r+Y_{1} \tilde{\theta}_{1}+Y_{2} \tilde{\theta}_{2}-k r-e_{v},
$$

where the notation $\tilde{\theta}_{2}(t) \in \mathbb{R}^{p_{2}}$ is defined as

$$
\tilde{\theta}_{2}=\theta_{2}-\hat{\theta}_{2} \text {. }
$$

Based on (23) and the subsequent stability analysis, the parameter estimates $\hat{\theta}_{1}(t)$ and $\hat{\theta}_{2}(t)$ are designed as

$$
\dot{\hat{\theta}}_{1}=\operatorname{proj}\left(\Gamma_{1} Y_{1}^{T} r\right) \quad \dot{\hat{\theta}}_{2}=\operatorname{proj}\left(\Gamma_{2} Y_{2}^{T} r\right),
$$

where $\Gamma_{1} \in \mathbb{R}^{p_{1} \times p_{1}}$ and $\Gamma_{2} \in \mathbb{R}^{p_{2} \times p_{2}}$ are constant, positivedefinite, diagonal adaptation gain matrices, and $\operatorname{proj}(\cdot)$ denotes a projection algorithm utilized to guarantee that the $i^{\text {th }}$ element of $\hat{\theta}_{1}(t)$ and $\hat{\theta}_{2}(t)$ can be bounded as

$$
\underline{\theta}_{1 i} \leq \hat{\theta}_{1 i} \leq \bar{\theta}_{1 i} \quad \underline{\theta}_{2 i} \leq \hat{\theta}_{2 i} \leq \bar{\theta}_{2 i},
$$

where $\underline{\theta}_{1 i}, \bar{\theta}_{1 i} \in \mathbb{R}$ and $\underline{\theta}_{2 i}, \bar{\theta}_{2 i} \in \mathbb{R}$ are known, constant lower and upper bounds for each element of $\hat{\theta}_{1}(t)$ and $\hat{\theta}_{2}(t)$, respectively.

\section{B. Adaptive Momentum Tracking Control Development}

Based on the tracking error of (14), the flywheel angular momentum tracking error can be quantified as

$$
\mu=h_{f}-I_{w s} \Omega(t) .
$$

For development of the closed-loop dynamics regarding the momentum tracking error, the time derivative of (40) is given as

$$
\dot{\mu}=-I_{w s} \dot{\Omega}(t) .
$$

Multiplying (41) by the known positive-definite symmetric matrix $I_{w s}^{-1}$, and substituting the first row of (34) into the resulting expansion yields

$$
I_{w s}^{-1} \dot{\mu}=R_{1}+N_{1} \bar{s}_{w} k_{w} g .
$$

Based on the structure of (42), the signal $g(t)$ is designed to satisfy the following relationship

$$
N_{1} \bar{s}_{w} k_{w} g=-R_{1}-k_{m} \mu,
$$

where $k_{m} \in \mathbb{R}$ is a positive constant control gain. Based on the Moore-Penrose pseudo-inverse property in (30a) and (30b), the minimum norm solution of (43) is given as

$$
g=\left(k_{w} N_{1} \bar{s}_{w}\right)^{-1}\left(-R_{1}-k_{m} \mu\right) .
$$

The result in (44) indicates that simultaneous attitude and momentum tracking is possible when $k_{w} N_{1} s_{w}$ is invertible. After substituting (44) into (42) for $g(t)$, the following closed-loop error system is obtained:

$$
I_{w s}^{-1} \dot{\mu}=-k_{m} \mu .
$$

\section{Stability Analysis}

Theorem 1: The weighted control input (27) including the flywheel acceleration and the gimbal rate along with the adaptive update laws given in (38) ensure global asymptotic attitude tracking such that

$$
\left\|e_{v}(t)\right\| \rightarrow 0 \quad \text { as } \quad t \rightarrow \infty
$$

and that (13) is satisfied, and then (8) is achieved, and exponential momentum tracking in the sense that

$$
\|\mu(t)\| \leq \mu_{0} \exp \left(-I_{w s} k_{m} t\right)
$$

where $\mu_{0} \in \mathbb{R}$ is a positive bounding constant.

Proof: The exponential momentum tracking result is evident from (45).

To prove the attitude tracking result, consider the nonnegative and radially unbounded function $V\left(e_{v}, e_{0}, r, \tilde{\theta}_{1}, \tilde{\theta}_{2}, t\right) \in$ $\mathbb{R}$ defined as

$$
\begin{aligned}
V \triangleq & e_{v}^{T} e_{v}+\left(1-e_{0}\right)^{2}+\frac{1}{2} r^{T} J r \\
& +\frac{1}{2} \tilde{\theta}_{1}^{T} \Gamma_{1}^{-1} \tilde{\theta}_{1}+\frac{1}{2} \tilde{\theta}_{2}^{T} \Gamma_{2}^{-1} \tilde{\theta}_{2} .
\end{aligned}
$$

Let $D \subset \mathbb{R}^{7}$ be a domain containing $y(t)=0$, where $y(t) \in$ $\mathbb{R}^{7}$ is defined as

$$
y(t) \triangleq\left[\begin{array}{ll}
z^{T}(t) & \sqrt{Q(t)}
\end{array}\right]^{T},
$$

and $z(t) \in \mathbb{R}^{6}$ is defined as

$$
z \triangleq\left[\begin{array}{ll}
e_{v}^{T} & r^{T}
\end{array}\right]^{T}
$$

The auxiliary function $Q(t) \in \mathbb{R}$ included in (49) is defined as

$$
Q(t) \triangleq \frac{1}{2} \tilde{\theta}_{1}^{T} \Gamma_{1}^{-1} \tilde{\theta}_{1}+\frac{1}{2} \tilde{\theta}_{2}^{T} \Gamma_{2}^{-1} \tilde{\theta}_{2},
$$

where the function $Q(t) \geq 0$ since $\Gamma$ is positive-definite. The defined Lyapunov function candidate satisfies the following inequalities:

$$
W_{1}(y) \leq V(y, t) \leq W_{2}(y)
$$

where the continuous positive definite fuctions $W_{1}(y)$, $W_{2}(y) \in \mathbb{R}$ are defined as

$$
W_{1}(y) \triangleq \frac{1}{2}\|y\|^{2}, \quad W_{2}(y) \triangleq\|y\|^{2},
$$


where $W_{1}(y), W_{2}(y) \in \mathbb{R}$ are continuous positive definite functions. After using (24), (36), and (37), the time derivative of $V(t)$ can be expressed as

$$
\begin{aligned}
\dot{V}= & e_{v}^{T}\left(e_{v}^{\times}+e_{0} I\right) \tilde{\omega}+\left(1-e_{0}\right) e_{v}^{T} \tilde{\omega} \\
& +r^{T}\left(Y_{1} \tilde{\theta}_{1}+Y_{2} \tilde{\theta}_{2}-k r-e_{v}\right) \\
& -\tilde{\theta}_{1}^{T} \Gamma_{1}^{-1} \dot{\hat{\theta}}_{1}-\tilde{\theta}_{2}^{T} \Gamma_{2}^{-1} \dot{\hat{\theta}}_{2} .
\end{aligned}
$$

By using (16), (38), and exploiting the fact that

$$
e_{v}^{T} e_{v}^{\times} \tilde{\omega}=0,
$$

the expression in (53) can be upper bounded as

$$
\dot{V} \leq-\lambda\|z\|^{2},
$$

where $\lambda=\lambda_{\min }\{\alpha, k\} \in \mathbb{R}$. The bounding result given in (54) achieves the following equivalent inequality:

$$
\dot{V} \leq-W(y),
$$

where $W(y)=c\|z\|^{2}$ is a continuous positive semi-definite function for a positive constant $c \in \mathbb{R}$. The Lyapunov function in (48) and the inequality in (55) can be used to conclude that $r(t), e_{v}(t), \tilde{\theta}_{1}(t), \tilde{\theta}_{2}(t) \in \mathcal{L}_{\infty}$. Thus, from (12), (16), and (50), $e_{v}(t), e_{0}(t), \tilde{\omega}(t), z(t) \in \mathcal{L}_{\infty}$, and (15) can be used to conclude that $\omega(t) \in \mathcal{L}_{\infty}$. Equation (9) then shows that $\dot{e}_{v}(t), \dot{e}_{0}(t) \in \mathcal{L}_{\infty}$. Exploiting $e_{v}(t), e_{0}(t), z(t) \in \mathcal{L}_{\infty}$, (21), (26), and (39) can be used to show that $g(t) \in \mathcal{L}_{\infty}$. The fact that $r(t), e_{v}(t), \tilde{\theta}_{1}(t), \tilde{\theta}_{2}(t) \in \mathcal{L}_{\infty}$ yields $\dot{r}(t) \in$ $\mathcal{L}_{\infty}$ from (36). From $\tilde{\theta}_{1}(t), \tilde{\theta}_{2}(t) \in \mathcal{L}_{\infty},(24)$, and (37), $\hat{\theta}_{1}(t), \hat{\theta}_{2}(t) \in \mathcal{L}_{\infty}$. Hence, (22), (26), (27), (31), (39), (44), and (47) can be used to prove that the control input $\dot{\eta}(t) \in$ $\mathcal{L}_{\infty}$. Standard signal chasing arguments can then be utilized to prove that all remaining signals remain bounded during closed-loop operation. Since $e_{v}(t), r(t), \dot{e}_{v}(t), \dot{r}(t) \in \mathcal{L}_{\infty}$, $e_{v}(t)$ and $r(t)$ are uniformly continuous. Therefore, $U(y)$ is uniformly continuous in $D$. Now, choose $d>0$ such that $B_{d} \subset D$, let $\rho<\min _{\|y\|=d} U_{1}(y)$, and consider the set $S$ satisfying $S \subset D \subset \mathbb{R}^{7}$ denoted as

$$
S \triangleq\left\{y(t) \in B_{d} \mid U_{2}(y) \leq \rho\right\} .
$$

Then, the result in (46) can now be obtained under all initial condition $y(0)$ in the set $S$ (i.e., $e_{v}(t) \rightarrow 0$ as $t \rightarrow \infty$, $\forall y(0) \in S)$.

\section{Simulation ANALYSiS}

The proposed controller was numerically simulated using the following dynamic parameters from the University of Florida (UF) CMG testbed: the total inertia $\left(J_{\text {total }}=\right.$ $\left.\operatorname{diag}\left\{6.10 \times 10^{-2} \quad 6.10 \times 10^{-2} \quad 7.64 \times 10^{-2}\right\}\right)$ and each 4-VSCMG unit mass $\left(m_{v s c m g}=0.165 \mathrm{~kg}\right)$ [14]. The simulation is started at an internal elliptic singularity configuration $\left(-90^{\circ}, 0^{\circ}, 90^{\circ}, 0^{\circ}\right)$, which is known as an inescapable singularity that cannot be avoided using null motion in conventional CMG system [15], [16]. The angular velocity $\omega(t)$ of the testbed tracks the desired angular velocity which is generated in $x$-axis and $z$-axis (after 100s) shown in Fig. 1. A sudden task assignment given in $z$-axis
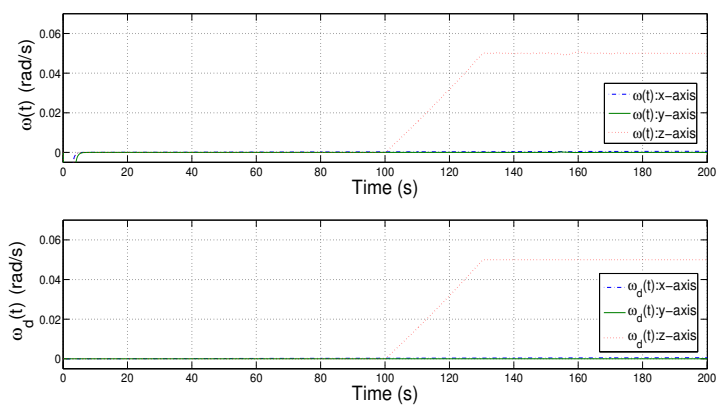

Fig. 1. Actual angular velocity $\omega(t)$ and desired angular velocity $\omega_{d}(t)$ during closed-loop operation from start of elliptic singularity.
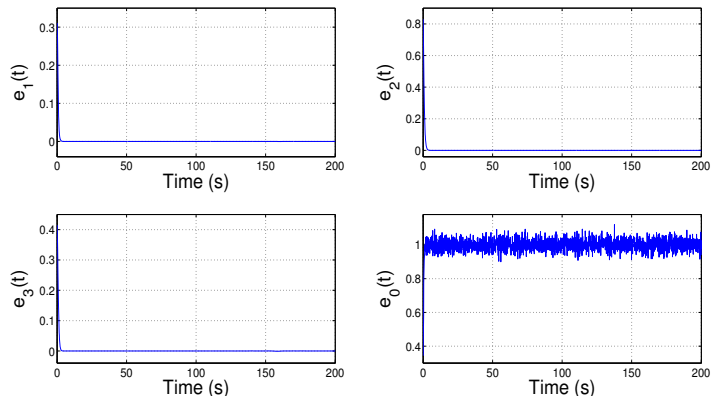

Fig. 2. Quaternion tracking error $e(t)$ during closed-loop operation from start of elliptic singularity.
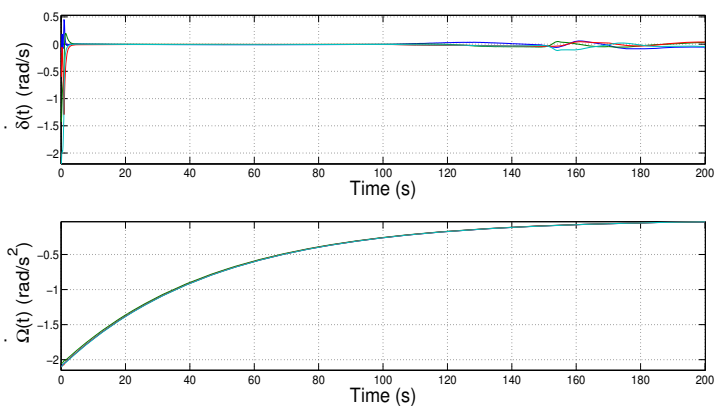

Fig. 3. Control input gimbal rates $\dot{\delta}(t)$ (top) and wheel accelerations $\dot{\Omega}(t)$ (bottom) from start of elliptic singularity.
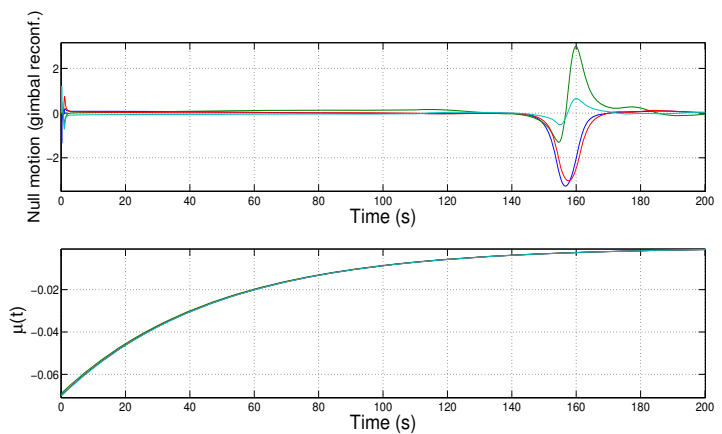

Fig. 4. Null motion: gimbal reconfiguration (top) and wheel speed regularization (bottom) from start of elliptic singularity. 

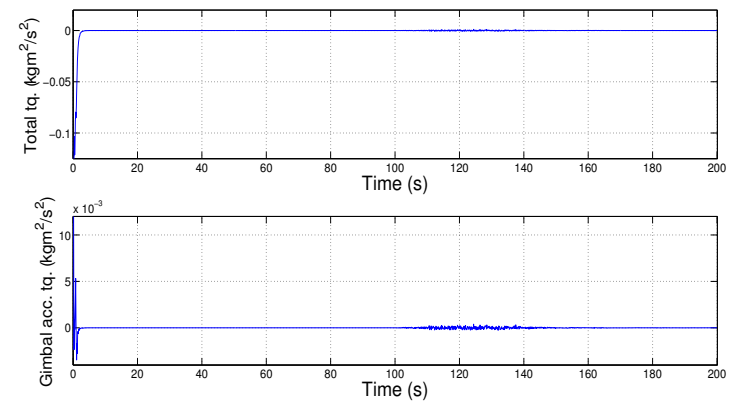

Fig. 5. Total torque and gimbal acceleration torque during closed-loop controller operation from start of elliptic singularity.

after $100 \mathrm{~s}$ is to evaluate the performance of the proposed controller in a severe situation. Figs. 2 - 5 show the simulation results of the closed-loop system when starting at the elliptic singularity. Specifically, Fig. 2 shows the quaternion tracking error. Fig. 3 shows the gimbal rate $\dot{\delta}(t)$ and the wheel acceleration $\dot{\Omega}(t)$ as the control inputs. The wheel acceleration control input $\dot{\Omega}(t)$ contributes to the momentum management by regulating the wheel speed to the preferred wheel speed $\Omega_{f}=200 \mathrm{rad} / \mathrm{s}(\approx 2,000 \mathrm{rpm})$. While the VSCMG system avoids an isolated point in the geometric configuration space by invoking RWs, the null motion of (31) depicted in Fig. 4 simultaneously brings about the gimbal reconfiguration as well as wheel speed regularizaion, which achieves the momentum management objective by making the momentum tracking error $\mu(t)$ of (14) approach zero as illustrated in Fig. 4. Fig. 5 shows the commanded total torque and the gimal acceleration torque which have been generally ignored as relatively small magnitudes comparing to the other torque terms. However, Fig. 5 shows that the magnitude of the gimbal acceleration torque with a unit of $\mathrm{kg} \cdot \mathrm{m}^{2} / \mathrm{s}^{2}$ is not trivial and provides about $10 \%$ of the total torque.

\section{CONCLUSIONS}

In this paper, an adaptive attitude controller for a VSCMGactuated satellite is presented. In the presence of uncertain, nonlinear, and time-varying inertia in the satellite, the controller is capable of achieving globally asymptotical attitude tracking while simultaneously performing singularity avoidance and momentum management by the null motion. The simulation shows that the controller as a VSCMG steering law is robust at start from elliptic singularity and even in sudden task assignment test. The wheel speed regularization resulting from the null motion achieves the momentum management which can help the system reduce power and hold the approach to the external saturation singularity. In addition, the controller compensates for the effects of uncertain, time-varying satellite inertia properties. The difficulty arising from uncertain satellite inertia is mitigated through an innovative development of the error system along with a Lyapunov-based adaptive law. The attitude tracking and momentum tracking results are proven via a Lyapunov stability analysis and demonstrated through numerical simulations.

\section{REFERENCES}

[1] C. Hall, "Integrated spacecraft power and attitude control systems using flywheels," Air Force Institute of Technology, Tech. Rep., 1997.

[2] H. Schaub, S. R. Vadali, and J. L. Junkins, "Feedback control law for variable speed control moment gyros," J. Astronaut. Sci., vol. 46, no. 3, pp. 307-328, Jul. 1998.

[3] H. Schaub and J. L. Junkins, "Singularity avoidance using null motion and variable-speed control moment gyros," J. Guid. Contr. Dynam., vol. 23 , no. 1, pp. 11-16, 2000.

[4] H. Kurokawa, "Survey of theory and steering laws of single-gimbal control moment gyros," Journal of Guidance, Control, and Dynamics, vol. 30, no. 5, pp. 1331-1340, 2007.

[5] H. Yoon and P. Tsiotras, "Spacecraft adaptive attitude and power tracking with variable speed control moment gyroscopes," J. Guid. Contr. Dynam., vol. 25, no. 6, pp. 1081-1090, Nov.-Dec. 2002.

[6] D. A. DeVon and R. J. Fuentes, "Adaptive attitude control and closedloop power tracking for an integrated power and attitude control system using variable speed control moment gyroscopes," in Proc. AIAA Guid. Navig. Control Conf., AIAA-2005-6394.

[7] D. J. Richie, V. J. Lappas, and G. Prassinos, "A practical small satellite variable-speed control moment gyroscope for combined energy storage and attitude control," in Proc. AAS/AIAA Astrodyn. Specialist Conf., Honolulu, HI, AIAA-2008-7503.

[8] J. Notti, A. Cormack, and W. Klein, "Integrated Power/Attitude Control System (IPACS)," J. Spacecraft Rockets, vol. 12, no. 5, pp. 485-491, 1975.

[9] R. Jansen, "Single axis flywheel IPACS@1300w, 0.8nm," in NASA Space Power Workshop, April 2005.

[10] D. Kim, W. MacKunis, N. Fitz-Coy, and W. E. Dixon, "Precision IPACS in the presence of dynamic uncertainty," in Proc. IEEE Conf. Decis. Control, Shanghai, China, Dec. 2009, pp. 5959-5964.

[11] W. E. Dixon, A. Behal, D. M. Dawson, and S. Nagarkatti, Nonlinear Control of Engineering Systems: A Lyapunov-Based Approach. Birkhäuser Boston, 2003.

[12] H. Yoon and P. Tsiotras, "Singularity analysis of variable-speed control moment gyros," J. Guid. Contr. Dynam., vol. 27, no. 3, pp. 374-386, 2004.

[13] B. Wie, D. Bailey, and C. Heiberg, "Singularity Robust Steering Logic for Redundant Single-Gimbal Control Moment Gyros," J. Guid. Contr. Dynam., vol. 24, no. 5, pp. 865-872, 2001.

[14] F. Leve, A. Tatsch, and N. Fitz-Coy, "A scalable control moment gyro design for attitude control of micro-, nano-, and pico-class satellites," in AAS Guid. Control Conf., Breckenridge, CO, 2007.

[15] B. Wie, "Singularity analysis and visualization for single-gimbal control moment gyros systems," J. Guid. Contr. Dynam., vol. 27, no. 2, pp. 271-282, March-April 2004.

[16] N. Bedrossian, J. Paradiso, E. Bergmann, and D. Rowell, "Redundant single gimbal control moment gyroscope singularity analysis," J. Guid. Contr. Dynam., vol. 13, no. 6, pp. 1096-1101, 1990. 